\title{
Natural diet of the crab Ovalipes catharus (Crustacea, Portunidae) around central and northern New Zealand
}

\author{
R. G. Wear \& M. Haddon \\ Island Bay Marine Laboratory, Department of Zoology, Victoria University of Wellington, Private Bag, Wellington, \\ New Zealand
}

\begin{abstract}
Foregut contents were examined of 4324 Ovalipes catharus (White 1843) collected from 11 locations in central and northern New Zealand, from November 1984 to September 1985. Of these 2572 foreguts contained recognizable food items, and a total of 72 species were identified in the diet. No significant differences were found in the relative proportions of foregut contents between males and females. Sand was an important component of the foregut contents, but was not considered part of the diet. When measured in terms of frequency of occurrence and estimated volumes (points method), Mollusca dominated the diet at 6 locations and Crustacea at 5. At all localities almost all the Mollusca eaten were small juvenile bivalves, mainly from the commercially and recreationally important genus Paphies. Callianassa filholi, $O$. catharus, isopods, and amphipods were important Crustacea in the diet at different localities. The diet at all localities differed significantly in the proportions of 14 dietary categories, but when 6 generalized dietary categories were considered similarities were found. $O$. catharus is a versatile and opportunistic predator. The possible impact of this predator on commercially important shellfish species is discussed.
\end{abstract}

\section{INTRODUCTION}

The paddle crab Ovalipes catharus (White 1843) occurs on sandy coastlines throughout New Zealand, the Chatham Islands, and in east and south Australia (Wear \& Fielder 1985). New Zealand coastlines extend $13000 \mathrm{~km}, 57 \%$ of which is sand-dominated $(0.063$ to $200 \mathrm{~mm}$ ) (J. G. Gibb, N. Z. Min. Works \& Devel., pers. comm.). Because of its abundance and large size (up to $140 \mathrm{~mm}$ carapace width) $O$. catharus is possibly the most significant invertebrate predator from the intertidal zone to about $100 \mathrm{~m}$ depth. It is also becoming increasingly important as a fishing resource (King 1985).

Along with recent increases of fishing effort and catches (with no perceptible decline in the resource), there is abundant anecdotal evidence of Ovalipes catharus biomass increasing throughout New Zealand (Wear 1982, Stead 1983). Its role as a predator may thus be changing

To assess how sandy-coast communities may be influenced by Ovalipes catharus, its natural diet was investigated. No previous analyses of the foregut contents of $O$. catharus have been published.
Commercial shellfisheries in Europe and North America are claimed to suffer serious damage through predation by the shore crab Carcinus maenas (Ropes 1968, Walne \& Dean 1972, Elner 1981, Dare et al. 1983). Shellfisheries of economic or recreational value at risk to predation by Ovalipes catharus in New Zealand are the 3 fisheries of the bivalve genus Paphies: pipi $P$. australis; tuatua $P$. subtriangulata; and toheroa $P$. ventricosa (Redfearn 1974, Wear 1984). Thus, a particular aspect of this present work was to assess the relative status of the 3 Paphies species and other bivalves of commercial and ecological importance in the natural diet of Ovalipes catharus.

\section{MATERIALS AND METHODS}

A total of 4324 crab foreguts from 11 sandy beach localities in central and northern New Zealand was examined (Fig. 1). Most crabs (2959) were from Raumati South, near Wellington.

Sampling methods. All crabs were collected using a $30 \mathrm{~m}$, heavily weighted seine net of $80 \mathrm{~mm}$ knot-toknot mesh, dragged by hand parallel to the shore in 


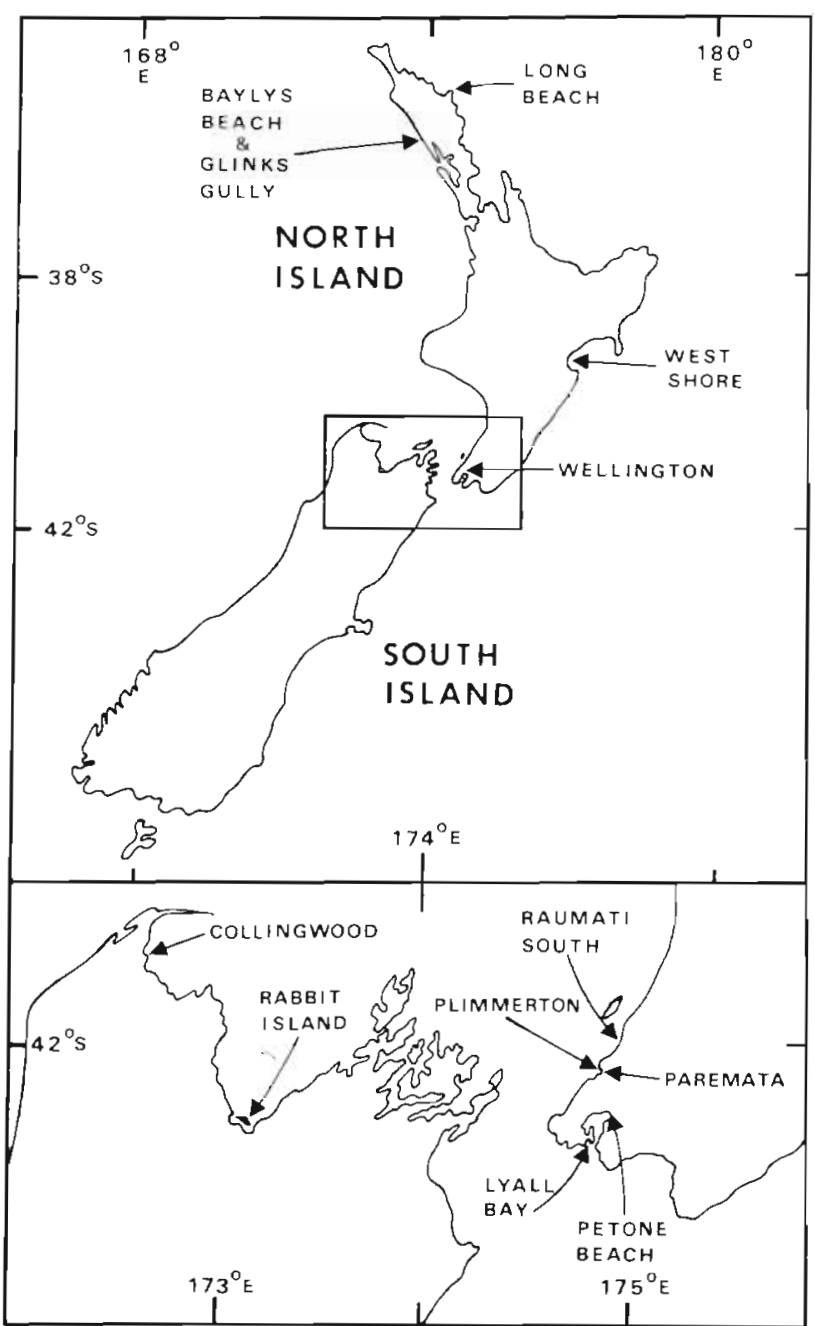

Fig. 1. Map of New Zealand with details of the Wellington area, showing the 11 sampling sites. Two sites (Glinks Gully and Baylys Beach) are located $15 \mathrm{~km}$ apart on Dargaville Beach

water 1.0 to $1.5 \mathrm{~m}$ deep. An exception was the Collingwood sample, which was made using a $110 \mathrm{~mm}$ knotto-knot mesh net. All samples were taken over low tide except for Raumati South, where in 10 of 12 sample sets, individual nettings at $6 \mathrm{~h}$ intervals over $24 \mathrm{~h}$ yielded approxımately equal numbers of crabs over both low and high tides. Sea conditions were calm or relatively calm, except during the Glinks Gully, Baylys Beach and West Shore samples, and for 9 of 43 separate nettings from Raumati South, for which wave heights were $\geqslant 1.0 \mathrm{~m}$.

All crabs sampled were picked from the net and their carapaces torn free from the body posteriorly to allow $10 \%$ formalin (in scawater) to penetrate immediately to the foregut. All crabs were preserved within $15 \mathrm{~min}$ of beaching. After a minimum $48 \mathrm{~h}$ fixation, each crab was sexed and carapace width was measured to the nearest $1.0 \mathrm{~mm}$. All crab foreguts were removed, their relative degree of fullness determined and each individually labelled and preserved for later analysis of contents.

Analysis of foregut contents. Relative degree of fullness was assessed visually and each foregut was placed in one of 6 ordered classes as follows: $100 \%$ full foreguts, designated Class 5; $75 \%(<100 \%$ but $>65 \%$ full), Class $4 ; 50 \%$ (<65\% but $>35 \%$ full), Class $3 ; 25 \%(<35 \%$ but $>5 \%$ full $)$, Class $2 ;<5 \%$ full or only a trace of food, Class 1 ; and empty foreguts, Class 0 . Foreguts $100 \%$ full were distinguishable as those in which the whole foregut was distended and the foregut wall tightly stretched. All foreguts were subsequently opened and any designated Class 1 $(<5 \%)$ found to be empty or to contain only liquid were recategorized as Class 0 . Any foreguts initially classed as empty and found to contain traces of food (occasionally caught in the gastric mill) were reclassified into Class 1

The use of only 6 classes of foregut fullness permitted different workers to classify separate collections of foreguts consistently. Visual assessment of fullness was made possible by the foregut of Ovalipes catharus being a thin-walled translucent bag, except for the gastric mill apparatus.

Five stages of digestion were determined under a dissecting microscope: (1) Identifiable soft and hard parts not separatedi soft material retaining natural colours. (2) Some soft material still adhering to identifiable hard parts. (3) Traces of identifiable soft material not attached to identifiable hard parts. (4) Unidentifiable, or no soft material, plus hard parts not readily identifiable. (5) Empty, or non-organic material, or containing only fluid. Only guts in Digestion Stages 1 to 3 were used in our analyses of foregut contents.

Foregut contents were identified to the lowest possible taxonomic level (Table 1), but for analysis contents were separated into 15 major categories ranging from the level of phylum to species (Tables $2 a, b$ ). Sand was included as a foregut content category but was not considered as a prey or a dietary category. Organic material included algal and terrestrial plant fragments and unidentifiable organic material. Major prey categories listed as species were the burrowing thalassinid shrimp Callianassa filholi, Ovalipes catharus (i.e. cannibalism), and the 3 bivalve molluscs Paphies australis, $P$. subtriangulata, and $P$. ventricosa. The 3 Paphies spp. were separated by shell texture and by hinge characteristics. The category 'Other Bivalves' includes identifiable bivalve species other than those of the genus Paphies, and also unidentifiable bivalve remains (which may therefore include any of the above species). Similarly, the category 'Other Crustacea includes those which were obviously not amphipods, isopods, or mysids, identifiable species other than 
those specified above, and also unidentifiable crustacean remains.

Points method. The relative contribution of the 15 foregut content categories, when present, to the total content of each foregut was subjectively assessed on a 5 step scale of points: A category representing 95 to $100 \%$ of foregut contents was given 100 points; $75 \%$ $(<95 \%$ and $>65 \%), 75$ points; $50 \% \quad(<65 \%$ and $>35 \%), 50$ points; $25 \%(<35 \%$ and $>5 \%), 25$ points $5 \%$ or less, 2.5 points. The number of points each category received was weighted according to the fullness of the foregut in which they were found. This weighting consisted of multiplying the number of points by a value dependent on the class of fullness: Class $5=1.0 ;$ Class $4=0.75$; Class $3=0.5$; Class $2=$ 0.25 ; Class $1=0.02$. Thus, the maximum weighted points a single category in a single foregut could obtain would be $100(100 \times 1.0$, i.e. only one category in a completely full foregut). The minimum possible would be $0.05(2.5 \times 0.02$, i.e. the category makes up $<5 \%$ of foregut contents where the gut is $<5 \%$ full).

Where data are expressed as percent frequency of occurrence these are based on the number of foreguts which contained food in Digestion Stages 1 to 3, and not on the total number of foreguts examined from a sample. Percent points was the proportion of the total number of points in the sample. Thus, the percent frequency of occurrence of any dietary category is independent of sand, but the percent points for a given category is dependent on whether sand is being considered.

Statistical analyses. To test for differences in the proportional constitution of foregut contents of the 2 sexes, at each of the 11 sampling localities, chisquared tests were performed on the frequency of occurrence of the 15 foregut content categories. These were combined when necessary to maintain expected frequencies greater than one.

To compare the proportional composition of the diets of crabs from the different sampling localities, chisquared tests, at the $5 \%$ level of significance, were performed on the frequency of occurrence of 6 generalized dietary categories. In the context of this work the 3 Paphies species were amalgamated into the first generalized category. The remaining 5 generalized categories for comparison were Crustacea, Mollusca, Organic Material, Fish, and Polychaeta. The general category Mollusca included all Paphies species, other bivalves, and all gastropods.

To obtain information on relative similarity of diet a multi-variate cluster analysis was performed on all 11 locations. To ensure that sample size had no influence on the clustering, the percent points of all the dietary categories were used as location properties. The 3 Paphies species were again considered together. Clus- tering was performed by the CLUSTER procedure from SAS using the average linkage criterion on the squared Euclidean distances (SAS Institute Inc. 1982).

To obtain a measure of how variable the diets from different locations were, the Shannon-Weiner diversity index of the diet category points was calculated using base 10 logarithms (Zar 1974) for each sampling locality.

\section{RESULTS}

From the total of 4324 foreguts examined 1094 $(25.3 \%)$ were empty, and of the $3230(74.7 \%)$ foreguts with contents, $2572(59.5 \%)$ contained material in Digestion Stages 1 to 3 . When all sampling localities were considered 72 different species were identified in the diet of Ovalipes catharus (Table 1).

No differences were found in the relative proportions of the foregut contents between males and females at any of the 11 sampling locations (all comparisons $p>0.1$ ). In the following analyses the data for both sexes are therefore combined.

\section{Frequency of occurrence and points}

Frequency of occurrence and total points for the 14 dietary categories, plus sand, for the 5 Wellington area sample locations, are given in Table $2 \mathrm{a}$. Those for the 6 remaining sampling locations are given in Table $2 \mathrm{~b}$. The relative proportions of the diet components, in terms of points (all categories except sand), are illustrated in Fig. 2 \& 3. Although sand was not considered part of the diet, it was, nevertheless, an important component of the foregut contents and contributed more than $20 \%$ to the total points at 6 locations ( $T a-$ bles $2 \mathrm{a}$, b): Plimmerton (51.7\%); Paremata (24.4\%); Rabbit Island $(30.9 \%)$; Glinks Gully $(44.5 \%)$; Baylys Beach $(62.1 \%)$; and West Shore $(41.8 \%)$.

In terms of points the most important generalized food category was either Crustacea or Mollusca dependent on location (Table 3). Thus, crustaceans were the most important component of the diet at Raumati South, Paremata, Lyall Bay, and Baylys Beach, whilst molluscs were the most important at Plimmerton, Petone, Rabbit Island, Collingwood, Glinks Gully, and West Shore. Long Beach (12 foreguts only) was an exception, with Crustacea, Mollusca, Organic Material, and Polychaeta all being of approximately the same relative importance (Table $2 b$ ).

In terms of frequency of occurrence the order of relative importance of Crustacea and Mollusca was the same as that found with the points system except at Paremata and Long Beach (Table 3). At Paremata the order is reversed in that molluscs appear more impor- 
Table 1. List of species in the diet of Ovalipes catharus. Locality abbreviations: RS, Raumati South; PL, Plimmerton; PA, Paremata; LY, Lyall Bay; PB, Petone Beach; RI, Rabbit Island; CO, Collingwood; DB, Dargaville Beach; WS, West Shore; LB, Long Beach. (Dargaville Beach includes both Baylys Beach and Glinks Gully; the same species were identifiable from both areas)

\begin{tabular}{|c|c|c|c|c|c|c|c|c|c|c|c|c|c|c|}
\hline Taxa & & & Locations & presen & & & & Taxa. & & & ncations & preser & & \\
\hline $\begin{array}{l}\text { Annelida } \\
\text { POLYCHAETA }\end{array}$ & & & & & & & & $\begin{array}{l}\text { Cirolanidae } \\
\text { Eurylana sircuate f tiatel }\end{array}$ & RS & & & & & $D B$ \\
\hline Arenicolidae & & & & & & & & Sphaeromatidae & 100 & & & & & \\
\hline Abarenicola sp. & RS & & PB & & & . & & Exosphaeroma obtusum (Dana) & & & LY & & & \\
\hline Glyceridae & & & & & & & & Exosphaeroma chilensis (Dana) & & & LY & & & \\
\hline Glycera sp. & RS & & PB & RI & & & & Sphaeromatidae (unidentified) & & & & RI & & DB \\
\hline Nephtyidae & & & & & & & & & & & & & & \\
\hline Algaophamus sp. & RS & & & & DB & & & AMPHIPODA & & & & & & \\
\hline Nereididae & & & & & & & & $\begin{array}{l}\text { Haustoriidte } \\
\text { Haustoridae (unidentified) }\end{array}$ & & & & & & \\
\hline $\begin{array}{l}\text { Nereididae (undidentified\} } \\
\text { Nereis ? falcaria \{Willey) }\end{array}$ & & & & & & & LB & $\begin{array}{l}\text { Haustoridae (unidentified) } \\
\text { Lysianassidae }\end{array}$ & RS & PL & & & & $\mathrm{DB}$ \\
\hline $\begin{array}{l}\text { Nereis ? falcaria (Willey) } \\
\text { Perinereis? brevicirris (Grube) }\end{array}$ & RS & & & & & & & $\begin{array}{l}\text { Lysianassidae } \\
\text { Lysianassidae (unidentified) }\end{array}$ & RS & & & & & \\
\hline $\begin{array}{l}\text { Perinereis ? brevicisris (Grube) } \\
\text { Onuphidae }\end{array}$ & & & PA & & & & & $\begin{array}{l}\text { Lysianassidae (unidentified) } \\
\text { Hyalidae }\end{array}$ & & & & & & \\
\hline Onuphidae (unidentified) & & & & & & & $L B$ & Hyalidae (unidentified) & RS & & & & & \\
\hline Onuphis sp. & & & & & & & LB & Isaeidae & & & & & & \\
\hline Opheliidae & & & & & & & & Isaeidae (unidentified) & RS & & & & & \\
\hline Euzonus otagoensis Probert & & & & & DB & & & Ampeliscidae & & & & & & \\
\hline Orbiniidae & & & & & & & & Ampeliscidae (unidentified) & RS & & & & & \\
\hline Orbiniidae (unidentified) & & & & & DB & & & Phoxocephalidae & & & & & & \\
\hline Pectinariidae & & & & & & & & Phoxocephalidae (unidentified) & RS & & & & & $\mathrm{DB}$ \\
\hline Pectinaria australis Ehlers & & & PB & & & & & Oedicerotidae & & & & & & \\
\hline Spionidae & & & & & & & & Patuki of breviuropodus & & & & & & $\mathrm{DB}$ \\
\hline Spionidae (unidentified) & & & & & DB & & & Cooper \& Fincham & & & & & & \\
\hline Scolelepis sp. & & & & $\mathrm{R} \perp$ & $D B$ & & & $\begin{array}{l}\text { Gammaridae } \\
\text { Gammaridae (unidentified) }\end{array}$ & $\mathrm{RS}$ & & & & & \\
\hline Mollusca & & & & & & & & $\begin{array}{l}\text { Gammaridae (unidentified) } \\
\text { Talitridae }\end{array}$ & KS & & & & & \\
\hline GASTROPODA & & & & & & & & Allorchestes novizealandiae Dana & RS & & LY & & & \\
\hline Trochidae & & & & & & & & & & & & & & \\
\hline Trochidae (unidentified) & RS & & & & & & & DECAPODA; THALASSINIDEA & & & & & & \\
\hline Cantharidus purpureus (Gmelnn) & RS & & LY & & & & & Callianassidae & & & & & & \\
\hline Cantharidella tesselata (A. Adams) & & & $\mathrm{LY}$ & & & & & Callianassa filhol A Milne Edwards & RS & & LY & & & DB \\
\hline $\begin{array}{l}\text { Micrelenchus dilatatus (Sowerby) } \\
\text { Eafoniellidae }\end{array}$ & & & LY & & & & & & & & & & & \\
\hline $\begin{array}{l}\text { Eatoniellidae } \\
\text { Eatoniella sp. }\end{array}$ & & & & & & & & $\begin{array}{l}\text { DECAPODA; ANOMURA } \\
\text { Paguridae }\end{array}$ & & & & & & \\
\hline $\begin{array}{l}\text { Eatoniella sp. } \\
\text { Calyptraeidae }\end{array}$ & & & PA LY & & & & & Paguristes barbatus (Heller) & RS & & PA & $\mathrm{RI}$ & & \\
\hline Zegalerus tenuis (Gray) & & & & & $\mathrm{DB}$ & & & 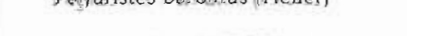 & (1) & & lis & & & \\
\hline Terebridae & & & & & & & & DECAPODA: BRACHYURA & & & & & & \\
\hline Duplicaria (Pervicacia) & RS & & & & & & & Portunidae & & & & & & \\
\hline tristis (Deshayes) & & & & & & & & Ovalipes catharus (White) & RS : & PL & PA LY & $\mathrm{RI}$ & & DB WS LB \\
\hline Fyramidellidae & & & & & & & & Majidae & & & & & & \\
\hline Turbonilla (Chemnitzia) sp. & RS & & & & & & & Notomithrax ursus (Herbst) & & & LY & & & \\
\hline BIVALVIA & & & & & & & & Pinnothendae & & & & & & \\
\hline $\begin{array}{l}\text { BIVALVIA } \\
\text { INuculidae }\end{array}$ & & & & & & & & Pinnotheres novaezealandae Filhol & RS & PL & PA & & & \\
\hline Nucula harivigiana Pfeiffer & & & PA & & & & & Hymenosomatidse & & & & & & \\
\hline Nucula nitidula A. Adams & RS & & PA & RI & & & & & & & $\begin{array}{l}\mathrm{PA} \\
\mathrm{PA}\end{array}$ & $\mathrm{PB}$ & & \\
\hline Mỵtilidae & & & & & & & & Hymenosoma depressum (Jaquinot) & RS & & & PB & & \\
\hline Mytulus edulis aoteanus Powell & RS & & LY & & $D B$ & & & Neohymenicus pubescens (Dana) & & & & PB & & \\
\hline Perna canaliculus (Gmelin) & $\mathrm{RS}$ & & PA LY & & DB & & & Grapsidae & & & & & & \\
\hline Mactridae & & & & & & & & Planes sp. & RS & & LY & & & \\
\hline Mactra discors Gray & RS & & & & & & & & & & & & & \\
\hline $\begin{array}{l}\text { Spisula (Crassula) } \\
\text { aequilaterahs (Deshayes) }\end{array}$ & RS & & LY & RI & & WS & & Echinodermata & & & & & & \\
\hline Mesodesniatidae & & & & & & & & ECHINOIDEA & & & & & & \\
\hline Paphies (Paphies) australis (Gmelin) & & & $\therefore \mathrm{PB}$ & & & & & Fellaster zelandiae (Gray) & RS & & & & & WS \\
\hline $\begin{array}{l}\text { Paphies (Paphies) } \\
\text { subtriangulata (Wood) }\end{array}$ & RS & PL & LY & RI & CODB V & ws & & OPHIUROIDEA & & & & & & \\
\hline Paphies (Paphies) ventricosa (Gray) & & & & & DB & & & Amphiura Spy & & PL & & & & \\
\hline Veneridae & & & & & & & & & & & & & & \\
\hline Dosinia sp & RS & PL. & & $\mathrm{RI}$ & CODB V & WS & & Pisces & & & & & & \\
\hline Tawera spissa (Deshayes) & & F & PA LY & & & & & Carangidae & & & & & & \\
\hline Austrovenus stutchburyi (Wood) & & & $\mathrm{PA}, \mathrm{PB}$ & : & $\therefore$ & & & Pseudocaranx dentex & RS & & & & & \\
\hline Crustacea & & & & & & & & (Bloch \& Schneider) & & & & & & \\
\hline COPEPODA. & & & & & & & & Clupeidae & & & & & & \\
\hline Thalestridae & & & & & & & & Sprattus sp. & RS & & & & & \\
\hline Thaiestridae (unidenuifiedj & RS & & & & & & & Engraulididae & & & & & & \\
\hline MYSIDACEA & & & & & & & & Engraulis australis (White) & RS & & & & & \\
\hline $\begin{array}{l}\text { MYSIDACEA } \\
\text { Lophogastridae }\end{array}$ & & & & & & & & Galaxidae & & & & & & \\
\hline & RS & & & & & & & Galaxias sp. & RS & & & & & \\
\hline $\begin{array}{l}\text { Tenagomysis of producta Tattersall } \\
\text { Tenagomysis macropsis Tattersall }\end{array}$ & & & & RI & & & & Mondae & & & & & & \\
\hline $\begin{array}{l}\text { Tenagomysis macropsis Tattersall } \\
\text { Tenagomysis ci. }\end{array}$ & RS & & & & & & & Pseudophycis bachus & RS & & & & & \\
\hline $\begin{array}{l}\text { Tenagomysis di. } \\
\text { aovae-zealandiae Thomson }\end{array}$ & & & & & & & & $\begin{array}{l}\text { (Bloch \& Schneider) } \\
\text { Leptoscopidae }\end{array}$ & & & & & & \\
\hline & & & & & & & & Crapatulus sp. & $\mathrm{RS}$ & & LY & & & \\
\hline ISOPODA & & & & & & & & Pleuronectidae & & & & & & \\
\hline Idoteidae & & & & & & & & Peftorhampus & RS & & & & & \\
\hline Zenobiana tubicola (Thomson) & RS & & LY & & & & & novdezeelandiae Gunther & & & & & & \\
\hline $\begin{array}{l}\text { Macrochiridothea uncinata } \\
\text { Hurley \& Murray }\end{array}$ & & & & & $\mathrm{DB}$ & & & $\begin{array}{l}\text { Tetraodontidae } \\
\text { Contusus richei (Freminvilie) }\end{array}$ & RS & & & & & \\
\hline
\end{tabular}


Table 2. Ovalipes catharus. Frequency of occurrence and points (see 'Materials and Methods') for the 15 foregut content categories from (a) the 5 Wellington area sample localities; (b) 6 sample localities outside Wellington area. Restricted to

Digestion Stages 1,2 , and 3

\begin{tabular}{|c|c|c|c|c|c|c|c|c|c|c|c|c|}
\hline \multicolumn{13}{|c|}{ (a) Wellington area } \\
\hline \multirow[t]{2}{*}{ Food category } & \multicolumn{2}{|c|}{$\begin{array}{l}\text { Raumati } \\
\text { South } \\
\text { all year }\end{array}$} & \multicolumn{3}{|c|}{$\begin{array}{l}\text { Plimmerton } \\
\text { Feb } 85\end{array}$} & \multicolumn{2}{|c|}{$\begin{array}{l}\text { Paremata } \\
\text { Feb } 85\end{array}$} & \multicolumn{3}{|c|}{$\begin{array}{l}\text { Lyall Bay } \\
\text { Dec } 84 \\
\text { Feb } 85\end{array}$} & \multicolumn{2}{|c|}{$\begin{array}{l}\text { Petone } \\
\text { Jan } 85\end{array}$} \\
\hline & Freq. & Pts & \multicolumn{2}{|c|}{ Freq. } & Pts & Freq. & Pts & Freq & \multicolumn{2}{|c|}{ Pts } & Freq. & Pts \\
\hline Sand & 1507 & 15746.2 & \multicolumn{2}{|r|}{58} & 1642.6 & 35 & 645.6 & 51 & \multicolumn{2}{|c|}{294.8} & 58 & 359.9 \\
\hline Organic material & 1165 & 14456.0 & \multicolumn{2}{|r|}{14} & 42.6 & 6 & 127.6 & 30 & \multicolumn{2}{|c|}{445.6} & 10 & 20.8 \\
\hline Callianassa filholi & 693 & 23530.0 & \multirow{2}{*}{\multicolumn{2}{|c|}{$\begin{array}{r}0 \\
24\end{array}$}} & 0.0 & 0 & 0.0 & 1 & \multicolumn{2}{|c|}{0.1} & 0 & 0.0 \\
\hline Ovalipes catharus & 284 & 8041.0 & & & 573.3 & 13 & 533.5 & 9 & \multicolumn{2}{|c|}{127.5} & 0 & 0.0 \\
\hline Mysidacea & 101 & 728.1 & \multicolumn{2}{|r|}{0} & 0.0 & 0 & 0.0 & 5 & 12 & 2.5 & 0 & 0.0 \\
\hline Isopoda & 407 & 6712.8 & \multicolumn{2}{|r|}{0} & 0.0 & 0 & 0.0 & 26 & 589 & & 0 & 0.0 \\
\hline Amphipoda & 251 & 1610.1 & & 1 & 3.8 & 0 & 0.0 & 15 & 346 & & 0 & 0.0 \\
\hline Other Crustacea & 408 & 6609.3 & & 13 & 67.2 & 27 & 677.4 & 15 & 325 & & 25 & 152.8 \\
\hline Paphies subtriangulata & 928 & 16415.0 & & 42 & 687.6 & 0 & 0.0 & 36 & 640 & & 0 & 0.0 \\
\hline P. ventricosa & 0 & 0.0 & & 0 & 0.0 & 0 & 0.0 & 0 & & 0.0 & 0 & 0.0 \\
\hline P. australis & 0 & 0.0 & & 0 & 0.0 & 0 & 0.0 & 0 & & 0 & 62 & 2165.5 \\
\hline Other bivalves & 518 & 7215.1 & & 16 & 34.0 & 42 & 647.3 & 7 & 80 & .4 & 14 & 335.3 \\
\hline Gastropoda & 4 & 33.8 & & 0 & 0.0 & 4 & 4.0 & 16 & 118 & & 0 & 0.0 \\
\hline Polychaeta & 12 & 219.6 & & 1 & 0.1 & 3 & 9.8 & 1 & 25 & 5.0 & 16 & 66.4 \\
\hline Fish & 266 & 7088.2 & & 4 & 128.8 & 0 & 0.0 & 3 & 65 & 5.0 & 2 & 3.0 \\
\hline N/Total & 1941 & 92659.0 & & 63 & 1537.4 & 66 & 1999.6 & 66 & 2780 & & 67 & 2743.8 \\
\hline (b) Other al & & & & & & & & & & & & \\
\hline Food category & $\begin{array}{c}\text { Rab } \\
\text { all }\end{array}$ & $\begin{array}{l}\text { bit Is. } \\
\text { year }\end{array}$ & $\begin{array}{l}\text { Collin } \\
\text { Fel }\end{array}$ & $\begin{array}{l}\text { gwood } \\
085\end{array}$ & $\begin{array}{r}\text { Gli } \\
\text { Jan, }\end{array}$ & $\begin{array}{l}\text { inks } \\
\text { Mar } 85\end{array}$ & $\begin{array}{r}\mathrm{Ba} \\
\mathrm{Jan},\end{array}$ & $\begin{array}{l}\text { ylys } \\
\text { Mar } 85\end{array}$ & $\begin{array}{l}\text { West } \\
\text { Feb }\end{array}$ & $\begin{array}{l}\text { Shore } \\
85\end{array}$ & $\begin{array}{r}\text { Long } \\
\text { Ja. }\end{array}$ & $\begin{array}{l}\text { Beach } \\
85\end{array}$ \\
\hline & Freq. & Pts & Freq. & Pts & Freq. & Pts & Freq & Pts & Freq. & Pts & Freq. & Pts \\
\hline Sand & 122 & 2173.3 & 15 & 90.2 & 101 & 2245.7 & 74 & 2297.8 & 24 & 598.2 & 10 & 85.4 \\
\hline Organic material & 22 & 128.9 & 0 & 0.0 & 18 & 91.2 & 20 & 186.3 & 2 & 6.3 & 3 & 170.0 \\
\hline Callianassa filholi & 0 & 0.0 & 0 & 0.0 & 0 & 0.0 & 0 & 0.0 & 0 & 0.0 & 0 & 0.0 \\
\hline Ovalipes catharus & 7 & 343.8 & 0 & 0.0 & 17 & 432.0 & 2 & 27.5 & 1 & 75.0 & 1 & 75.0 \\
\hline Mysidacea & 2 & 37.5 & 0 & 0.0 & 0 & 0.0 & 0 & 0.0 & 1 & 25.0 & 0 & 0.0 \\
\hline Isopoda & 2 & 53.8 & 0 & 0.0 & 9 & 68.0 & 11 & 87.6 & 0 & 0.0 & 4 & 6.2 \\
\hline Amphipoda & 1 & 12.5 & 0 & 0.0 & 15 & 88.4 & 16 & 235.1 & 0 & 0.0 & 0 & 0.0 \\
\hline Other Crustacea & 60 & 1387.3 & 7 & 162.5 & 22 & 211.6 & 27 & 315.3 & 5 & 78.8 & 2 & 56.4 \\
\hline Paphies subtriangulata & 73 & 1665.4 & 12 & 261.2 & 35 & 620.0 & 2 & 10.0 & 14 & 446.0 & 2 & 3.8 \\
\hline P. ventricosa & 0 & 0.0 & 0 & 0.0 & 14 & 60.5 & 27 & 230.4 & 0 & 0.0 & 0 & 0.0 \\
\hline$P$. australis & 0 & 0.0 & 0 & 0.0 & 0 & 0.0 & 0 & 0.0 & 0 & 0.0 & 0 & 0.0 \\
\hline Other bivalves & 57 & 1049.8 & 3 & 41.3 & 39 & 850.7 & 24 & 164.0 & 13 & 100.9 & 1 & 71.3 \\
\hline Gastropoda & 1 & 0.1 & 0 & 0.0 & 1 & 0.1 & 4 & 11.3 & 0 & 0.0 & 1 & 75.0 \\
\hline Polychaeta & 2 & 6.3 & 0 & 0.0 & 21 & 372.8 & 7 & 127.3 & 1 & 100.0 & 3 & 146.3 \\
\hline Fish & 4 & 163.8 & 0 & 0.0 & 1 & 2.5 & 4 & 7.1 & 0 & 0.0 & 3 & 113.5 \\
\hline $\mathrm{N} /$ Total & 128 & 4849.2 & 16 & 465.0 & 112 & 2797.8 & 76 & 1401.9 & 25 & 832.0 & 12 & 717.5 \\
\hline
\end{tabular}

tant than crustaceans, but this reversal is equivocal as Crustacea and Mollusca occur in approximately equal numbers of stomachs $(40: 43)$. At Long Beach, in terms of frequency of occurrence, Crustacea are the most important dietary item followed by Mollusca (Table 3).

\section{Dietary components}

Whilst it was possible to conclude whether Crustacea or Mollusca was more important at different locations, the specific dietary components which dominated these 2 generalized categories differed in most cases (Tables $2 \mathrm{a}, \mathrm{b}$ ).

At all localities except Paremata and Long Beach, a high proportion of the Mollusca eaten were members of the genus Paphies (Table 3). The species of Paphies eaten, and their relative importance in the diet, reflected availability. Thus, $P$. subtriangulata (tuatua) was common in most samples, but at Glinks Gully, which is close to a large bed of $P$. ventricosa (toheroa), toheroa was more important than tuatua (Table $2 b$, 

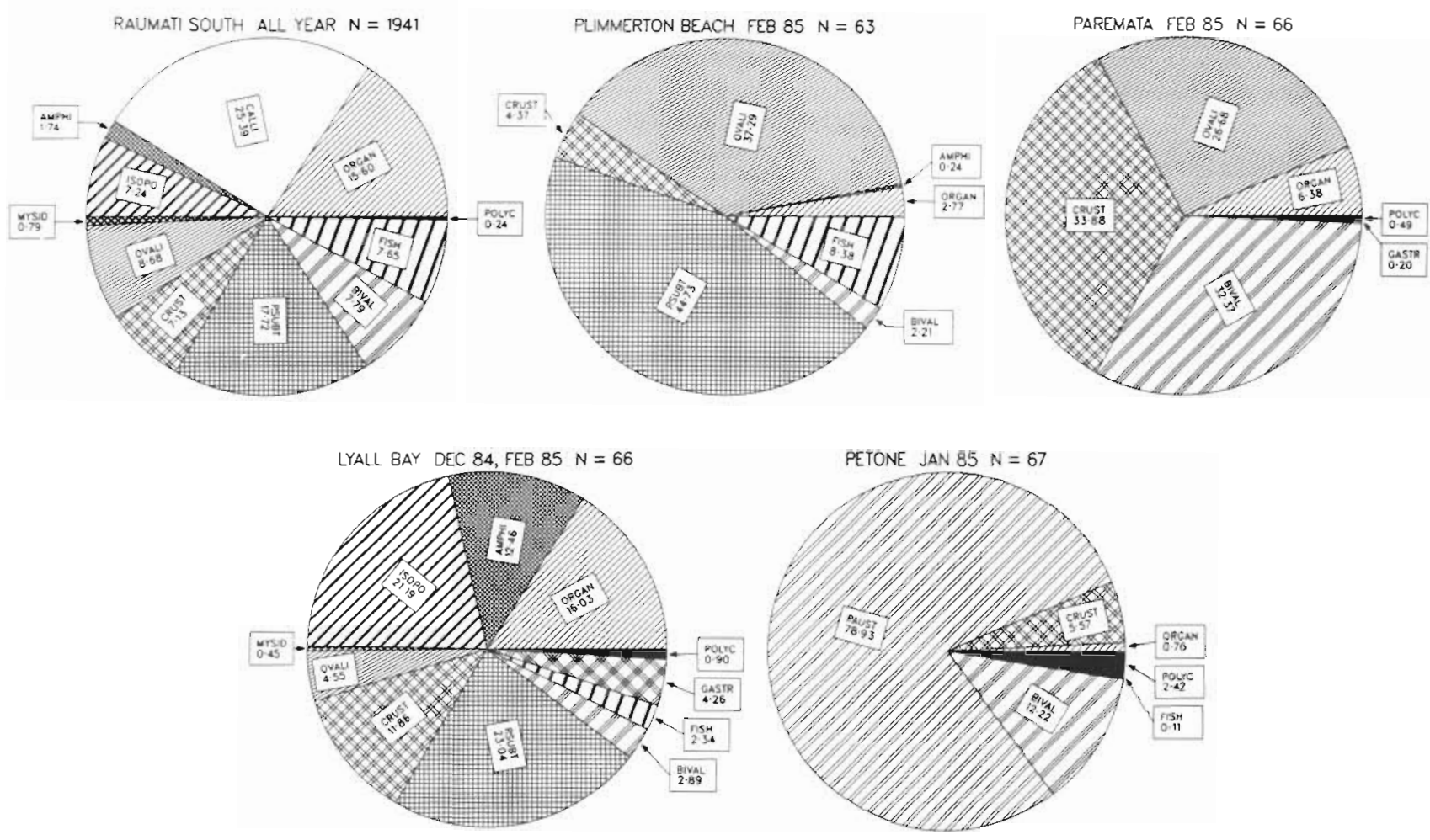

Fig. 2. Ovalipes catharus. Percent points of the 14 dietary categories for the 5 Wellington area sampling sites. N: number of foreguts with contents at Digestion Stages 1 to 3
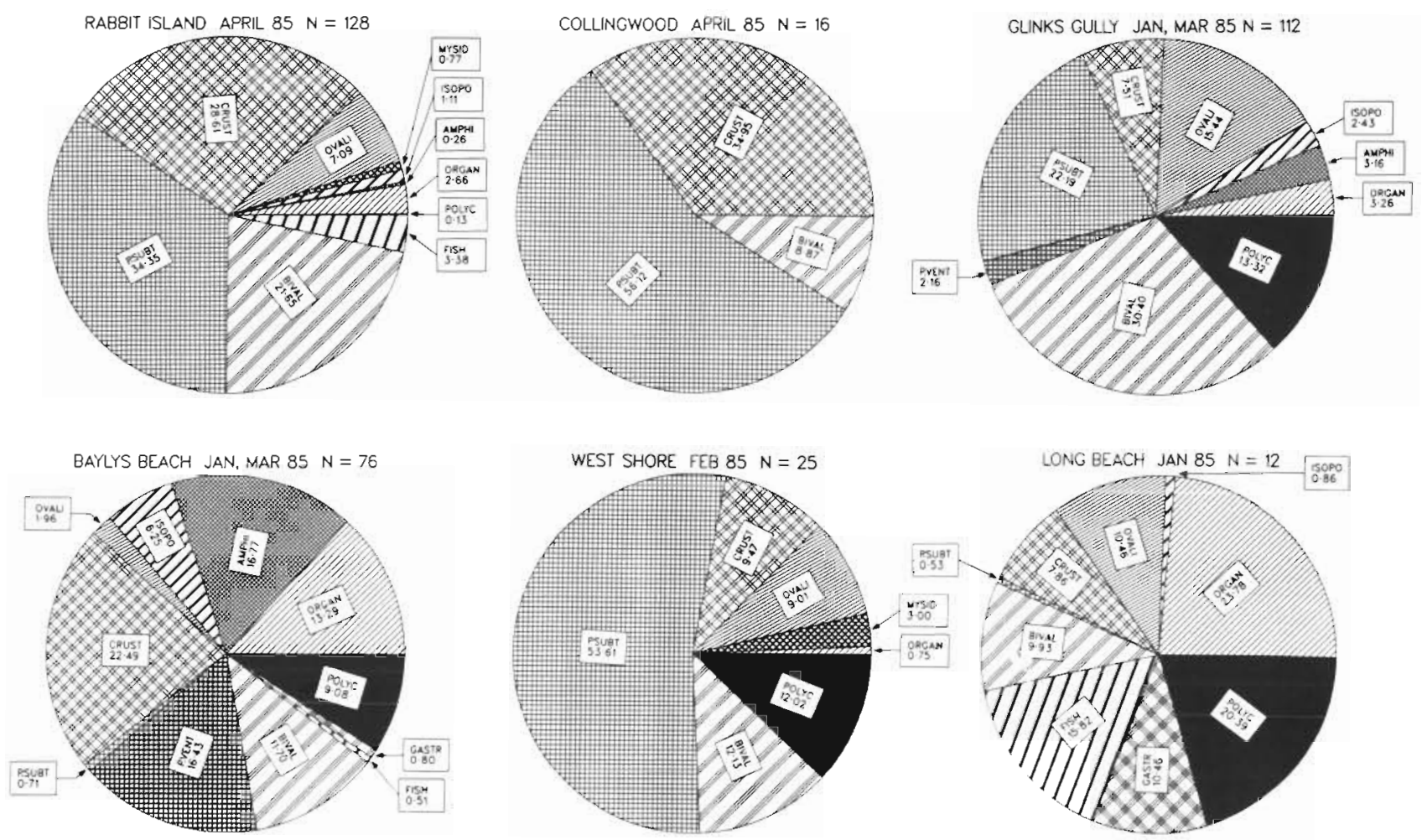

Fig. 3. Ovalipes catharus. Percent points of the 14 dietary categories for the 6 sampling sites beyond the Wellington area. $N$ : number of foreguts with contents at Digestion Stages 1 to 3 
Table 3. Percent points (see 'Materials and Methods') and percent frequency of occurrence of 3 generalized food categories. Crustacea, Mollusca, and the molluscan genus Paphies, in the diet of Ovalipes catharus from 11 central and northern New Zealand localities

\begin{tabular}{|c|c|c|c|c|c|c|c|}
\hline \multirow[t]{2}{*}{ Location } & \multirow[t]{2}{*}{$N$} & \multicolumn{3}{|c|}{ Points } & \multicolumn{3}{|c|}{ Frequency } \\
\hline & & $\%$ Crustacea & $\%$ Mollusca & $\%$ Paphies & $\%$ Crustacea & $\%$ Mollusca & $\%$ Paphies \\
\hline Raumati South & 1941 & 50.97 & 25.54 & 17.72 & 81.66 & 61.98 & 47.81 \\
\hline Plimmerton & 63 & 41.91 & 46.94 & 44.72 & 58.73 & 82.54 & 66.67 \\
\hline Paremata & 66 & 60.56 & 32.57 & 0.00 & 60.61 & 65.15 & 0.00 \\
\hline Lyall Bay & 66 & 50.54 & 30.20 & 23.04 & 81.82 & 72.73 & 54.55 \\
\hline Petone & 67 & 5.57 & 91.14 & 78.92 & 37.31 & 100.00 & 92.54 \\
\hline Rabbit Island & 128 & 37.84 & 55.99 & 34.34 & 56.52 & 81.25 & 57.03 \\
\hline Collingwood & 16 & 34.95 & 65.05 & 56.17 & 43.75 & 81.25 & 75.00 \\
\hline Glinks Gully & 112 & 28.59 & 54.73 & 24,32 & 50.00 & 66.07 & 43.75 \\
\hline Baylys Beach & 76 & 47.47 & 29.65 & 17.15 & 63.16 & 61.84 & 38.16 \\
\hline West Shore & 25 & 21.49 & 65.73 & 53.61 & 28.00 & 88.00 & 56.00 \\
\hline Long Beach & 12 & 19.18 & 20.92 & 0.53 & 50.00 & 33.33 & 16.67 \\
\hline
\end{tabular}

Fig. 3). Also, at Petone, a harbour location where only $P$. australis is found, this species was very important in the diet and neither of the other 2 open coast species of Paphies occur (Table 2a, Fig. 2). At Long Beach $P$. subtriangulata forms only a very minor part of the diet. Finally, at Paremata, although $P$. australis is present, the genus Paphies was not represented in the diet; the bivalve most frequently eaten was Nucula hartvigiana (Table 1).

Other than the 3 Paphies species, other bivalve species in the diet which are of commercial or recreational value included: blue mussel Mytilus edulis aoteanus; green-lipped mussel Perna canaliculus; and New Zealand cockle Austrovenus stutchburyi. All bivalve species were usually eaten by Ovalipes catharus at a very small size. Most shell fragments and hinges derived from bivalves less than 3 to $4 \mathrm{~mm}$ shell length. Indeed, crab foreguts packed with shell fragments often contained more than 100 hinges. Gastropoda were important in the diet only at Long Beach where the sample consisted of only 12 crabs (Tables $2 a$, b). Polychaeta were important at Glinks Gully, Baylys Beach, West Shore, and Long Beach (Table 2b, Fig. 3). Their importance elsewhere was not underestimated since digestion-resistant setae and jaws were present and influenced the frequency of occurrence, which, for Polychaeta, provides the same pattern of relative importance in different places as do points (Table $2 \mathrm{a}$, Fig. 2).

Burrowing ghost shrimp Callianassa filholi was the most important crustacean in the diet at Raumati South, but occurred nowhere else except in a single crab from Lyall Bay. Ovalipes catharus, however, was present in its own diet at all localities except Petone and Collingwood where only 'Other Crustacea' were present. Further, $O$. catharus was the most important component of Crustacea at Plimmerton, Glinks Gully, and Long Beach, and the second most important crustacean component at Raumati South, Paremata, Rabbit Island, and West Shore. 'Other Crustacea' was the most important component of Crustacea at Paremata, Rabbit Island, Baylys Beach, and West Shore, and was the only component at Petone and Collingwood. At Petone, 'Other Crustacea' (= Crustacea at this locality) was mostly the small spider crab Halicarcinus whitei, although other species did occur (Table 1). At Collingwood, however, all crustacean remains were unidentifiable. Of the remaining 3 major categories of Crustacea, Mysidacea were not important at any locality; Isopoda were relatively important only at Lyall Bay; and Amphipoda were important only at Baylys Beach (Tables 2a, b). Most isopods and amphipods present in the diet are species normally associated with drift algae (G. R. F. Hicks, National Museum of N.Z., pers. comm.). All occurrences of the pea crab Pinnotheres novaezealandiae, found at Raumati South, Paremata, and Plimmerton (Table 1), were small, free-living males rather than females which are commensal in bivalve molluscs. This species is thus considered an actual dietary item, rather than a species incidentally ingested with bivalve flesh.

\section{Comparison of diet with location}

There are 55 comparisons possible when the diet of Ovalipes catharus at each location is compared with every other location. The relationships between localities, in terms of the relative proportions of the 6 generalized dietary categories, were very similar to those found by cluster analysis of percent points (Table 4, Fig. 4). The main differences are at Paremata and Long Beach, both of which were significantly different from all other locations (Table 4, Fig. 2 to 4). This is 
Table 4. Ovalipes catharus. Chi-squared comparisons of the frequency of occurrence of 6 generalized diet categories. $\mathrm{Chi}^{2}$ $>11.07$ indicates significant differences. Only the 9 localities which included non-significant differences are listed

\begin{tabular}{|lrrrrrrr|}
\hline Location & $\begin{array}{c}\text { Lyall } \\
\text { Bay }\end{array}$ & $\begin{array}{c}\text { Rab- } \\
\text { bit } \\
\text { Is- } \\
\text { land }\end{array}$ & $\begin{array}{r}\text { Col- Glinks Baylys } \\
\text { ling- Gully }\end{array}$ & & & & \\
& & & & & \\
& & & & & & & \\
\hline Raumati South & 8.95 & 69.59 & 20.52 & 342.52 & 88.69 & 35.14 \\
Plimmerton & 8.29 & 1.39 & 5.20 & 16.42 & 9.54 & 6.17 \\
Lyall Bay & & 13.66 & 11.75 & 24.45 & 6.90 & 15.35 \\
Petone & 29.32 & 6.33 & 13.73 & 28.26 & 4.10 \\
Rabbit Island & & & 4.79 & 23.21 & 14.91 & 5.43 \\
Collingwood & & & & 9.00 & 12.29 & 3.05 \\
Glinks Gully & & & & & 10.02 & 8.30 \\
\hline
\end{tabular}

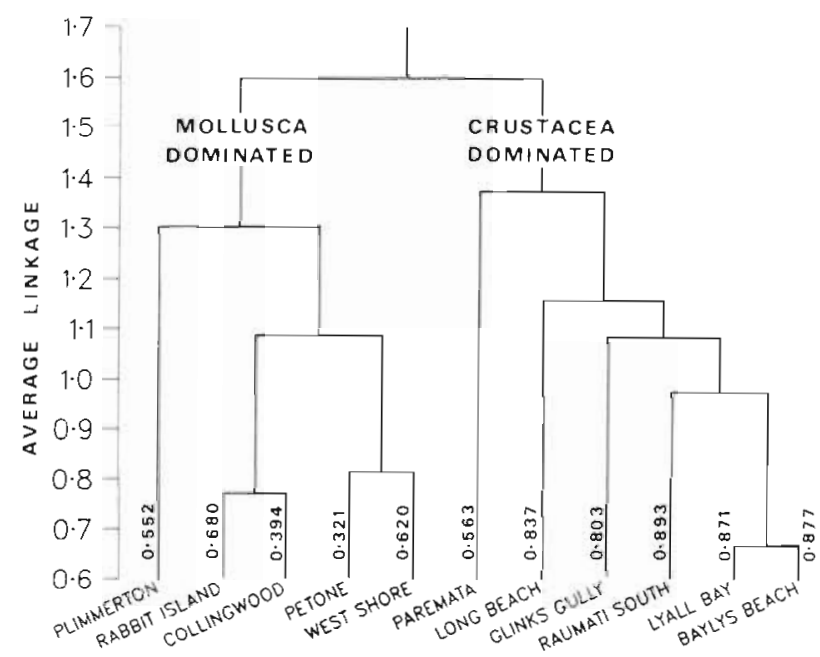

Fig. 4. Cluster diagram expressing the similarity of dietary composition in terms of percent points between locations. The Shannon-Weiner diversity index is given at the root of each branch. Despite the diet at Glinks Gully being dominated by Mollusca, the high diversity associated it with the Cnustacea dominated localities

reflected in their being the last 2 members to join the right-hand cluster. The diet at Glinks Gully is not significantly different from Baylys Beach but nor is it different from either Collingwood or West Shore. This reflects the fact that the diet at Glinks Gully is dominated by Mollusca. Of the molluscan-dominated diet group, Plimmerton is the only equivocal member in terms of $\mathrm{chi}^{2}$. The analysis shows that Plimmerton is not significantly different from members of both major groupings (Table 4). Its position as the last member of the molluscan grouping reflects this fact.

The cluster analysis (Fig. 4) indicates which locations are similar with respect to proportional make-up of the diet (Fig. $2 \& 3$ ). Although the chi-squared analysis of the proportional representation in the diet of the 6 generalized categories is not a test of the cluster analysis, it does provide corroboration that the clusters are meaningful. The diet of Ovalipes catharus at the 5 localities grouped on the left of the dendrogram (Plimmerton, Rabbit Island, Collingwood, Petone, and West Shore) was dominated by Mollusca. Also, the diversities of these 5 samples were less than 0.69 . The group on the right of the dendrogram (Paremata, Long Beach, Glinks Gully, Raumati South, Lyall Bay, and Baylys Beach) is more complex. This grouping reflects either a dietary diversity greater than 0.80 , or a diet dominated by Crustacea, or both. Thus, Baylys Beach. Lyall Bay, and Raumati South have highly diverse diets dominated by Crustacea. These 3 localities also form the basis of the whole group. In order of addition to this primary group there are: Glinks Gully which has a highly diverse diet (0.8029) dominated by Mollusca; Long Beach which has a diet of high diversity, but where Crustacea are only equal dominant (Fig. 3); and Paremata which has a djet of low diversity but is dominated by Crustacea.

Plimmerton and Raumati South are geographically close (Fig. 1) but are distant on the dendrogram, and Lyall Bay and Baylys Beach are geographically distant but are closely linked on the diagram (Fig. 4). Thus, the groupings in Fig. 4 do not reflect geographical proximity except in the case of Rabbit Island and Collingwood.

In terms of subjective estimates of sampling site exposure, the first 4 sites of the crustacean-dominated grouping were the most exposed of all sites. The least exposed sites amongst this cluster (Long Beach and Paremata) were the last members to be added to the group; the sites with diets dominated by Mollusca were relatively more sheltered. Thus, the degree of exposure to wave action is reflected, to some extent, in dietary composition (Fig. 4).

\section{Comparison of biological properties by location}

The size range of crabs caught was 18 to $134 \mathrm{~mm}$ carapace width (Table 5). In general, the size range of all samples was at least between 30 and $95 \mathrm{~mm}$ carapace width except at Collingwood, Glinks Gully, and Baylys Beach. At Collingwood the larger mesh size used to make the collection explains why crabs less than $56 \mathrm{~mm}$ carapace width are not represented in the sample. On Dargaville Beach (i.e. Glinks Gully and Baylys Beach sites), however, despite exhaustive sampling, no crabs greater than $79 \mathrm{~mm}$ carapace width were caught (Table 5). This may be related to the extreme exposure of this coastline. If rough sea conditions interfere with feeding then crabs on Dargaville Beach would often experience periods of reduced feeding. 
The sex ratio varied from $1: 12.3$ (males: females) at Baylys Beach, to $1: 0.47$ at Long Beach. However, females predominated at all localities except Long Beach, Rabbit Island, and Collingwood (Table 5). The fishery for crabs is mainly for the larger males which are found offshore in up to $100 \mathrm{~m}$ of water. It would appear that male crabs do not spend as much time in shallow beach waters as females and possibly are onshore in numbers only for breeding or when immature. As the diet of the 2 sexes was not found to differ, this partial segregation in space could possibly act to reduce the potential for competition.

\section{DISCUSSION}

Our analysis of foregut contents indicates that, in New Zealand, Ovalipes catharus is an opportunistic, generalist predator. At different locations the particular composition of the diet was found to differ but was generally either predominantly Mollusca or Crustacea (Table 3). At all localities except Long Beach, the molluscan component of the diet was almost completely composed of bivalves, most of which were, when available, one of the 3 species of Paphies (Tables $2 a, b \& 3$ ). The diversity of the diet also varied with location, with the highest diversities being found generally in diets dominated by Crustacea (Table 5 , Fig. 4). This variability between locations indicates that to characterize the diet of a widespread, active, predatory crab by considering animals caught only at one location or one time period may be misleading.

Although the fauna of New Zealand sandy coasts is documented in general texts (Morton \& Miller 1973), details of all the prey species presently available at our 11 sample locations are not known. At Dargaville
Beach, several species noted as dominant or common (Redfearn 1974) occurred in the diet of Ovalipes catharus. For example, polychaetes were recorded in dense aggregations just below mid-tide level (Redfearn 1974, authors obs.) and were found in the diets at both Glinks Gully and Baylys Beach (Fig. 3). The diets from these 2 locations on Dargaville Beach differed in terms of the relative proportions of tuatua Paphies subtriangulata and toheroa $P$. ventricosa. At Glinks Gully few toheroa could be found intertidally but there was a dense bed of juvenile tuatua on the upper shore. Conversely, at Baylys Beach toheroa were abundant intertidally and tuatua rare. The proportions of these 2 species in the diet at the 2 localities reflected their relative abundance. The diet of $O$. catharus at other localities also reflected availability of prey species. Thus, at Raumati South both the diet (Fig. 2) and the infauna (pers. obs.) was dominated by burrowing ghost shrimp Callianassa filholi and by $P$. subtriangulata. At Paremata, Nucula hartvigiana is very common on Zostera seagrass flats (Powell 1979), and occurred frequently in the diet; the cockle Austrovenus stutchburyi has been reported to reach densities up to $2500 \mathrm{~m}^{-2}$ (Healy 1980), and may shield deeper burrowing $P$. australis (Fig. 2). At Petone, dense beds of $P$. australis occur whilst cockles are relatively sparse, and this is also reflected in the diet (Fig. 2).

The diverse species composition of the diet (Table 1) demonstrates that Ovalipes catharus is extremely versatile in its feeding behaviour. Crabs are able to catch mobile fish, mysid shrimps, amphipods, isopods, and frequently to capture Callianassa filholi up to about $50 \mathrm{~mm}$ total length. The small degree of trituration, and the relative ease with which we were able to identify newly ingested prey, indicated that most were alive and in good condition when captured. Cannibal-

Table 5. Ovalipes catharus. Properties of all crab samples taken from the 11 locations

\begin{tabular}{|c|c|c|c|c|c|c|c|c|c|}
\hline Location & $\begin{array}{c}\text { Total } \\
\text { caught }\end{array}$ & Date & $\begin{array}{l}\text { Temp. } \\
\left({ }^{\circ} \mathrm{C}\right)\end{array}$ & $\begin{array}{l}\text { Sex } \\
\text { ratio } \\
M: F\end{array}$ & $\begin{array}{l}\% \\
\text { Guts } \\
\text { empty }\end{array}$ & $\begin{array}{l}\% \\
\text { Guts } \\
\text { full }\end{array}$ & $\begin{array}{c}\% \\
\text { Guts } \\
\text { dig. } 1-3\end{array}$ & $\begin{array}{c}\text { Diversity } \\
\text { of } \\
\text { diet }\end{array}$ & $\begin{array}{c}\text { Size } \\
\text { range } \\
(\mathrm{mm})\end{array}$ \\
\hline Raumati South & 2959 & 5 Nov $84-30 \operatorname{Sep} 85$ & $10-24$ & $1: 4.32$ & 19.5 & 65.6 & 17.0 & 0.8930 & $27-134$ \\
\hline Plimmerton & 106 & 22 Feb 85 & 19 & $1: 2.53$ & 24.5 & 10.4 & 60.4 & 0.5521 & $34-112$ \\
\hline Paremata & 126 & 22 Feb 85 & 18 & $1: 1$ & 30.2 & 5.6 & 52.3 & 0.5639 & $33-95$ \\
\hline Lyall Bay & 147 & $3-4$ Dec 84 & 18 & $1: 1.53$ & 42.9 & 11.6 & 44.9 & 0.8712 & $30-110$ \\
\hline Petone & 72 & $23 \operatorname{Jan} 85$ & 21 & $1: 1.4$ & 4.2 & 12.5 & 93.1 & 0.3209 & $45-98$ \\
\hline Rabbit Island & 330 & $25-27$ Apг 85 & $13-14.5$ & $1: 0.84$ & 46.7 & 6.7 & 38.8 & 0.6803 & $18-120$ \\
\hline Collingwood & 82 & 4 Apr 85 & - & $1: 0.86$ & 52.4 & 0.0 & 19.5 & 0.3936 & $56-122$ \\
\hline Glinks Gully & 250 & $\begin{array}{l}13-14 \text { Jan } 85 \\
4 \text { Mar } 85\end{array}$ & $20-22$ & $1: 9.0$ & 37.6 & 4.4 & 44.8 & 0.8029 & $20-79$ \\
\hline Baylys Beach & 159 & $\begin{array}{l}13-14 \operatorname{Jan} 85 \\
4 \text { Mar } 85\end{array}$ & $20-22$ & $1: 12.3$ & 34.6 & 9.4 & 47.8 & 0.8773 & $23-75$ \\
\hline West Shore & 71 & 26 Feb 85 & 19 & $1: 2.38$ & 47.9 & 11.3 & 35.2 & 0.6198 & $27-116$ \\
\hline Long Beach & 22 & $9-10$ Jan 85 & 24 & $1: 0.47$ & 31.8 & 18.2 & 54.6 & 0.8370 & $18-108$ \\
\hline
\end{tabular}


ism appeared common in nature, and in the laboratory, crabs of all sizes were vulnerable at ecdysis (pers. obs.; see also Elner 1981). In experiments O. catharus could locate and excavate bivalves buried in sand (Haddon et al. 1987). They also use their chelipeds to repeatedly shovel or scoop sand containing very small bivalves into the mouthparts. This latter method of feeding, combined with the importance of small bivalves in the diet (even where Crustacea-dominated), possibly explains the high relative importance of sand in foregut contents (Tables 2a, b).

It is possible that the differences observed between localities are due only to differences of sampling date. However, Glinks Gully and Baylys Beach were sampled either over the same low tide, or $1 \mathrm{~d}$ apart, and Plimmerton and Paremata were sampled within $1 \mathrm{~h}$ of each other. The Glinks Gully site is approximately $15 \mathrm{~km}$ from Baylys Beach but the respective diets, although similar (Fig. 4), are dominated by different species (Table $2 \mathrm{~b}$ ). Plimmerton is only approximately $1 \mathrm{~km}$ from Paremata but the diets at these 2 localities were very different from each other. This evidence suggests that geographic location and habitat determine the general availability of potential prey categories, but availability of particular prey species may also vary seasonally.

The natural diet of other crab species, including other species of Ovalipes, has been investigated and all have been found to be opportunist predators (Ropes 1968, Caine 1974, Elner 1981, Morales \& Antezana 1983, Du Preez 1984, Haefner 1985). The major components of the diet of $144 \mathrm{O}$. stephensoni from the continental shelf ( 13 to $198 \mathrm{~m}$ depth) off the eastern United States were crustaceans (especially amphipods and isopods), bivalve molluscs, and squid (Haefner 1985). Without stating how many foreguts were examined, Caine (1974) found that the diet of $O$. guadulpensis (but see Haefner 1985), on the Florida Gulf coast, was composed mainly of fish, ophiuroids, crustaceans, and annelids, with molluscs being found only occasionally. Again without stating the number of forcguts examined, Du Preez (1984), working in South Africa, found that $O$. punctatus fed mainly on gastropod and bivalve molluscs. Whilst the major items of the diet differ with geographic location, all authors agreed that the Ovalipes species they studied were generalist or opportunistic predators. Ropes (1968) and Elner (1981), who both studied large samples of Carcinus maenas, found that the diet was dominated by bivalve molluscs and considered that $C$. maenas would therefore be a pest on commercial shellfisheries.

It is probable that Ovalipes catharus has little direct impact on established beds of mature shellfish comprising species of commercial or recreational importance in New Zealand. Although we observed large numbers of bivalve shells chipped in a manner suggesting crab predation (Blundon \& Kennedy 1982, Du Preez 1984, Wear 1984) at both Raumati South and Rabbit Island, these may have accumulated over long periods. Adult Paphies ventricosa populations are protected to some extent by long periods of exposure during mid to low tides, and by depth of burial and high density acting as refuges from crab predation (Haddon et al. 1987). P. subtriangulata and $P$. australis greater than $40 \mathrm{~mm}$ shell length are resistant to crushing (Wear 1984), and adult cockles may be protected by their thick and highly inflated shells (Boulding 1984). Natural mussel populations are established on hard substrates where $O$. catharus does not occur in large numbers. However, $O$. catharus may influence recruitment among the bivalves discussed above, and others listed in Table 1, through heavy predation on spat and small individuals, as most foreguts with mollusc contents included large numbers of bivalve spat rather than mollusc flesh attributable to larger bivalves.

Acknowledgements. We thank Ms. Sarah Crane and Messrs Hugh Packer, Mark Goldman, and George Grainger for technical assistance; Mr. K. J. King for the Collingwood crab sample; and Dr. Derek and Mrs. Teresa Gibbons for kindly providing us with laboratory and collecting facilities at Dargaville Beach. We acknowledge the following National Museum of New Zealand staff for assistance with identifying crab gut contents: Dr. A. N. Baker (echinoderms); Dr. G. R. F. Hicks (isopods, amphipods, mysids); Mr. C. Paulin (fishes); Mr. B. A. Marshall (molluscs). Dr. C. B. Read and Mr. C. Roberts assisted us in identifying polychaetes and fishes respectively. Financial support for the research was provided by the Golden Kiwi Lottery Scientific Research Committee and the Victoria University Internal Research Committee. Dr. M. Haddon was supported during the study period by a University Grants Committee postdoctoral fellowship awarded by Victoria University of Wellington.

\section{LITERATURE CITED}

Blundon, J. A., Kennedy, V. S. (1982). Refuges for infaunal bivalves from blue crab. Callinectes sapidus (Rathbun), predation in. Chesapeake Bay. J. exp. mar Biol. Ecol. 65: $67-81$

Boulding, E. G. (1984). Crab-resistant features of shells of burrowing bivalves: decreasing vulnerability by increasing handling time. J. exp. Mar. Biol. Ecol. 76: 201-224

Caine, E. A. (1974). Feeding of Ovalipes guadulpensis (Saussurel (Decapoda: Brachyura: Portunidae), and morphological adaptations to a burrowing existence. Biol. Bull. mar. biol. Lab., Woods Hole 147 550-559

Dare, P. J., Davies, G., Edwards, D. B. (1983). Predation on juvenile Pacific oysters (Crassostrea gigas Thunberg) and mussels (Mytilus edulis L.) by shore crabs (Carcinus maenas L.). U.K. Min. Agr. Fish. and Food Fish. Res. Tech. Rep. 73: 1-15

Du Preez, H. H. (1984). Molluscan predation by Ovalipes punctatus (De Haan) (Crustacea: Brachyura: Portunidae). J. exp. mar. Biol. Ecol. 84: 55-71 
Elner, R. W. (1981). Diet of green crab Carcinus maenas (L.) from Port Hebert, southwestern Nova Scotia. J. Shellfish Res. 1: 89-94

Haddon, M., Wear, R. G., Packer, H. (1987). Depth of burial and density in the bivalve Paphies ventricosa as refuges from crab predation. Mar Biol (in press).

Haefner, P. A. (1985). Morphometry, reproduction, diet, and epizoites of Ovalipes stephensoni Williams, 1976 (Decapoda, Brachyura). J. Crust. Biol. 5: 658-672

Healy, W B. (1980). Pauatahanui Inlet - an environmental study. N.Z. Dept. Sc. Ind. Res. D.S.I.R. Information Ser. 141: $1-198$

King, M. R. (1985). Fish and shellfish landings by domestic fishermen, 1974-82. N.Z. Min. Agr. Fish., Fish. Res. Div. Occ. Publ. 20: 1-122

Morales, C., Antezana, T. (1983). Diet selection in the Chilean stone crab Homolaspis plana. Mar. Biol. 77: 79-83

Morton, J. E., Miller, M. C. (1973). The New Zealand sea shore. 2nd edn. Collins, Auckland

Powell, A. W. B. (1979). New Zealand Mollusca. Marine, land and freshwater shells. Collins, Auckland
Redfearm, P. (1974). Biology and distribution of the Toheroa, Paphies (Mesodesma) ventricosa (Gray). N.Z. Min. Agr. Fish., Fish. Res. Bull. 11-1-51

Ropes, J. W. (1968). The feeding habits of the green crab, Carcinus maenas (L.). Fish. Bull. U.S. 67: 183-203

SAS Institute Inc. (1982). SAS user's guide: Statistics. SAS Institute Inc., Cary, North Carolina

Stead, D. (1983). Paddle crab investigations. Catch '83, 3: $14-15$

Walne, P. R., Dean, G. J. (1972). Experiments on predation by the shore crab, Carcinus maenas L. on Mytilus and Mercenaria J. Cons. int. Explor. Mer 34: 190-199

Wear, R. G. (1982). Paddle crabs: a potential industry or a pest? Shellfish Newslet. 16. Suppl. to Catch '82, 11: 16-17

Wear, R. G. (1984). Paddle crabs are probable predators. Shellfish Newslet. 22, Suppl. to Catch '84, 5: 11-13

Wear, R. G., Fielder, D. R. (1985). The marine fauna of New Zealand: larvae of the Brachyura (Crustacea, Decapoda). N.Z. Oceanogr. Inst. Mem. 92: 1-90

Zar, J. H. (1974). Biostatistical analysis. Prentice Hall, London

This article was presented by Professor J. D. Costlow, Jr.; it was accepted for printing on October 29, 1986 